Chirurg 2021 · 92:350-360 https://doi.org/10.1007/s00104-020-01267-8 Online publiziert: 2 . September 2020 (c) Der/die Autor(en) 2020

\section{Anmerkung der Autoren}

Die Autoren möchten darauf hinweisen, dass dieser Artikel nicht als Leitlinie, sondern vielmehr als evidenzbasierter Expertenkonsens nach bester Evidenz und systematischer Literatursuche zu verstehen ist. Daher sei an dieser Stelle nochmals explizit auf die in diesem Zusammenhang existierende aktuelle S3-Leitlinie verwiesen [1].

\section{Hintergrund}

Die chirurgische Resektion ist die zentrale kurative Therapiemodalität für nichtmetastasierte Ösophaguskarzinome. Insbesondere die Inzidenz des Adenokarzinoms des Ösophagus ist in den letzten zwei Dekaden ganz erheblich gestiegen und ist eine der am stärksten zunehmenden Krebserkrankungen in Europa und Nordamerika. Das klassische Plattenepithelkarzinom des Ösophagus macht ebenso einen weiterhin relevanten

Jens Hoeppner ${ }^{1}$ für Qualitätskommission der Deutschen Gesellschaft für Allgemein- und Viszeralchirurgie · Patrick Sven Plum² für Qualitätskommission der Deutschen Gesellschaft für Allgemein- und Viszeralchirurgie · Heinz Buhr ${ }^{3}$ für Qualitätskommission der Deutschen Gesellschaft für Allgemein- und Viszeralchirurgie • Ines Gockel ${ }^{4}$ für Qualitätskommission der Deutschen Gesellschaft für Allgemein- und Viszeralchirurgie · Dietmar Lorenz ${ }^{5}$ für Qualitätskommission der Deutschen Gesellschaft für Allgemein- und Viszeralchirurgie - Michael Ghadimi ${ }^{6}$ für Qualitätskommission der Deutschen Gesellschaft für Allgemein- und Viszeralchirurgie · Christiane Bruns ${ }^{2}$ für Qualitätskommission der Deutschen Gesellschaft für Allgemein- und Viszeralchirurgie

' Klinik für Allgemein- und Viszeralchirurgie, Universitätsklinikum Freiburg, Medizinische Fakultät, AlbertLudwigs-Universität Freiburg im Breisgau, Freiburg, Deutschland; ${ }^{2}$ Klinik für Allgemein-, Viszeral-, Tumorund Transplantationschirurgie, Universitätsklinikum Köln, Köln, Deutschland; ${ }^{3}$ Deutsche Gesellschaft für Allgemein- und Viszeralchirurgie, Berlin, Deutschland; ${ }^{4}$ Klinik für Viszeral-, Thorax-, Transplantations- und Gefäßchirurgie, Universitätsklinikum Leipzig, Leipzig, Deutschland; ${ }^{5}$ Chirurgische Klinik I, Allgemein-, Viszeral- und Thoraxchirurgie, Klinikum Darmstadt, Darmstadt, Deutschland; ${ }^{6}$ Klinik für Allgemein-, Viszeral- und Kinderchirurgie, Universitätsmedizin Göttingen, Göttingen, Deutschland

\title{
Chirurgische Therapie des Ösophaguskarzinoms - Qualitätsindikatoren für Diagnostik und Therapie
}

Teil der chirurgischen und kurativ behandelbaren Fälle aus. Durch diagnostische, chirurgisch technische und perioperative Verbesserungen konnten Morbidität und Mortalität der anspruchsvollen Ösophagusresektion in den letzten 20 Jahren deutlich verringert werden. Die onkologische Langzeitprognose der Erkrankung nach Resektion konnte durch Einführung neoadjuvant radiochemotherapeutischer und perioperativ chemotherapeutischer Behandlungsstrategien ebenfalls deutlich verbessert werden.

Die Autoren haben im Rahmen der Qualitätsinitiative der Deutschen Gesellschaft für Allgemein- und Viszeralchirurgie (DGAV) eine Übersichtsarbeit auf der Grundlage einer systematischen Literatursuche verfasst und Empfehlungen für die aktuelle Diagnostik und Therapie des Ösophaguskarzinoms erarbeitet.

\section{Material und Methoden}

Die systematische Literatursuche erfolgte gemäß den PRISMA-Kriterien (Preferred Reporting Items for Systematic Reviews and Meta-Analyses) unter Verwendung der MEDLINEDatenbank (https://www.ncbi.nlm.nih. gov/pubmed/) und wurde im März 2019 durchgeführt [2]. Die dabei zu betrachtenden Themenkomplexe („perioperative multimodale onkologische Therapiekonzepte“, „perioperatives Management“, „stadienabhängige kurative Therapie und Operationsindikation " und „Tumordiagnostik bei Primärstaging und Restaging, Krankenhausstruktur“) wurden in jeweils eigenen Suchabfragen zusammengefasst und anschließend hieraus die relevanten Publikationen extrahiert. Um eine ausreichende Aktualität der Daten zu gewährleisten, wurde ein Suchzeitraum vom 01.01.2010 bis zum 13.03.2019 gewählt. Die gewählten Suchbegriffe entsprachen dabei den PRESS- 
Kriterien (Peer Review of Electronic Search Strategies).

Insgesamt wurden initial anhand der Kriterien 6879 Artikel identifiziert. Es konnten 5588 Duplikate nachgewiesen werden, sodass für die weiteren Analysen 1291 Artikel zur Verfügung standen. Nach Sichtung des Titels und/oder des Abstracts wurden letztlich 255 Artikel für die Volltextuntersuchung genutzt, von denen wiederum 25 Texte als Metaanalysen exkludiert wurden. Für die vorliegende Metaanalyse wurden final 230 Originalarbeiten herangezogen.

\section{Mindestmengen und Zentrali- sierung der Ösophaguschirurgie}

Die in Deutschland geltende Mindestmenge von 10 komplexen Ösophagusresektionen/Jahr/Krankenhaus ist im europäischen Vergleich all der Länder, in denen Mindestmengen gelten, die niedrigste. Positive Auswirkungen einer Zentralisierung lassen sich an unterschiedlichen Parametern festmachen, wie die niederländische Qualitätsinitiative „Dutch Upper Gastrointestinal Cancer Audit“ (DUCA) eindrucksvoll belegte. Zwischen 2011 und 2014 wurde dieses webbasierte Qualitätsprogramm für die Therapie der Ösophagus- und Magenkarzinome eingeführt [3]. Für beide Tumorentitäten wurde die jährliche Mindestmenge auf 20 Resektionen/Jahr festgelegt. Im letzten Jahr der Einführung wurden knapp $100 \%$ der operierten Patienten erfasst: Über den Beobachtungszeitraum sank die Krankenhausletalität von $8 \%$ auf $4 \%$ (Magenkarzinom) und blieb bei ca. $4 \%$ stabil (Ösophaguskarzinom). Für das Ösophaguskarzinom hatten allerdings schon vorher Mindestmengen gegolten. In Dänemark wurde bei Eingriffen am Ösophagus die Anzahl der operierenden Institutionen 2006 von 26 auf nur 4 reduziert wurde. Die Krankenhausletalität wurde dadurch von $4,5 \%$ auf $1,7 \%$ gesenkt [4].

Schon für die in Deutschland geltende Mindestmenge von 10 Ösophagusresektionen/Jahr/Institution wurde in einer Analyse der Arbeitsgruppe um Mansky ein signifikanter Überlebensunterschied festgestellt. Danach lag die Krankenhausletalität in den Häusern <10/Jahr bei $12,1 \%$ und für die $>10 / \mathrm{Jahr}$ bei $9,2 \%$ [5]. Anhand der deutschlandweiten Krankenhausabrechnungsdaten (DRG[„diagnosis related groups“]-Statistik) von 2010 bis 2015 wurde die Mengen-Ergebnis-Beziehung in Bezug auf die Krankenhaussterblichkeit, die Häufigkeit von Komplikationen und die Sterblichkeit von Patienten, bei denen Komplikationen auftraten, analysiert [6]. Insgesamt wurden 22.700 Behandlungsfälle mit komplexen Ösophaguseingriffen identifiziert. In Krankenhäusern mit sehr hoher Fallzahl (im Median $62 \mathrm{Be}$ handlungsfälle pro Jahr) bestand im Vergleich zu Krankenhäusern mit sehr geringer Fallzahl (im Median 2 Behandlungsfälle pro Jahr) eine halb so große Wahrscheinlichkeit, infolge des Ösophaguseingriffs $\mathrm{zu}$ versterben (Odds Ratio [OR]:0,50;95\%-Konfidenzintervall [KI]: 0,42; 0,60). Die Krankenhaussterblichkeit der Patienten mit Komplikation lag in Krankenhäusern mit sehr hoher Fallzahl bei $12,3 \%$, in Krankenhäusern mit sehr geringer Fallzahl bei 20,0\%.

In der deutschen Statistik wurden alle komplexen Ösophagusresektionen erfasst - also auch Notfalleingriffe, z. B. wegen Perforation. Insofern sind die absoluten Zahlen nicht mit denen aus den Niederlanden oder Dänemark vergleichbar, wo nur elektive Resektionen dokumentiert wurden. Das Prinzip ist jedoch das gleiche: Komplikationen nach Ösophagusresektionen sind häufig [4] und die Möglichkeit, Patienten aus diesen kritischen Situationen zu retten, hängt von der Erfahrung („volume“) und der Ausstattung eines Zentrums ab. Ist beides nicht in ausreichendem Maß vorhanden, versterben die Patienten an den Folgen der Komplikationen signifikant häufiger („failure to rescue“).

\section{Tumordiagnostik}

Die exakte Diagnostik des Ösophaguskarzinoms ist unabdingbar für die Therapieentscheidung. Neben der Anamnese und der körperlichen Untersuchung sind die Ösophagogastroduodenoskopie (ÖGD) mit Biopsie, der endoskopische Ultraschall (EUS) und die Computertomographie (CT) die drei wichtigsten Untersuchungstechniken in der Diagnostik.

\section{Ösophagogastroduodenoskopie mit Biopsie}

Durch die hochauflösende ÖGD wird die direkte Tumorvisualisierung und Lokalisierung mit Höhen- und Größenangabe und die Entnahme von Biopsien ermöglicht. Es sollte weiterhin eine zirkuläre Lokalisationsangabe, der Stenosierungsgrad sowie die Höhe des oberen Ösophagussphinkters und der Z-Linie bzw. des Zwerchfelldurchtritts dokumentiert werden. Die ÖGD mit Biopsie erreicht die höchste Sensibilität und Spezifität für einen Tumornachweis im oberen Gastrointestinaltrakt. Die Methode ist breit verfügbar und zeigt Komplikationsraten von $1 \%$ und eine Letalität $<0,1 \%$ [7]. Biopsien sollten immer aus allen verdächtigen Läsionen entnommen und getrennt asserviert und beurteilt werden. Die Ausdehnung einer evtl. vorhandenen Barrett-Metaplasie sollte ebenfalls inklusive Biopsie erfasst werden. Sofern endoskopisch-makroskopisch der Verdacht auf einen malignen Prozess besteht, aber eine negative Histologie vorliegt, sollte eine Reendoskopie mit multiplen Rebiopsien erfolgen.

Zur Verbesserung der HD-WeißlichtEndoskopie (HD-WLE) existieren Verfahren, die die Sensitivität verbessern sollen. Beschrieben sind die Chromoendoskopie, die computerbearbeitete virtuelle Chromoendoskopie („narrow-bandimaging“"u.a.) und die Vergrößerungsendoskopie (Endomikroskopie). Eine Metaanalyse, welche 11 randomisierte kontrollierte Studien (RCTs) einschlossen, untersuchte, ob die klassischen und die virtuellen Chromoendoskopieverfahren die Ergebnisse der HDWLE bei der Aufdeckung von Ösophagusneoplasien beim Barrett-Ösophagus verbessen können. Dabei verbesserten die Chromoendoskopie und die virtuellen Verfahren die bioptisch überprüfte Aufdeckungsraten von HG-IEN (hochgradige intraepitheliale Neoplasie) und Frühkarzinomen um bis $\mathrm{zu} 34 \%$ (KI $20-56 \%, p<0,0001$; [8]). Ein Einsatz dieser Verfahren wird daher von der deutschen Leitlinie empfohlen. 
Chirurg 2021 · 92:350-360 https://doi.org/10.1007/s00104-020-01267-8

(c) Der/die Autor(en) 2020

\begin{abstract}
J. Hoeppner für Qualitätskommission der Deutschen Gesellschaft für Allgemein- und Viszeralchirurgie · P. S. Plum für Qualitätskommission der Deutschen Gesellschaft für Allgemein- und Viszeralchirurgie · H. Buhr für Qualitätskommission der Deutschen Gesellschaft für Allgemein- und Viszeralchirurgie - I. Gockel für Qualitätskommission der Deutschen Gesellschaft für Allgemein- und Viszeralchirurgie · D. Lorenz für Qualitätskommission der Deutschen Gesellschaft für Allgemein- und Viszeralchirurgie · M. Ghadimi für Qualitätskommission der Deutschen Gesellschaft für Allgemein- und Viszeralchirurgie · C. Bruns für Qualitätskommission der Deutschen Gesellschaft für Allgemein- und Viszeralchirurgie
\end{abstract}

\title{
Chirurgische Therapie des Ösophaguskarzinoms - Qualitätsindikatoren für Diagnostik und Therapie
}

\section{Zusammenfassung}

Hintergrund. Im Rahmen der Qualitätsinitiative der Deutschen Gesellschaft für Allgemeinund Viszeralchirurgie (DGAV) wurde eine Übersichtsarbeit auf der Grundlage einer systematischen Literatursuche verfasst und Empfehlungen für die aktuelle Diagnostik und Therapie des Ösophaguskarzinoms erarbeitet. Methoden. Die systematische Literatursuche erfolgte gemäß den PRISMA-Kriterien unter Verwendung der MEDLINE-Datenbank und wurde im März 2019 durchgeführt. Die Empfehlungen wurden auf der Grundlage von Abstimmungen in der DGAV formuliert. Ergebnisse und Schlussfolgerung Operationen unterhalb der derzeit gül- tigen Mindestmenge sollten nicht mehr durchgeführt werden. Es spricht vieles dafür, die Mindestmenge in Deutschland auf $\geq 20$ Resektionen/Jahr/Krankenhaus anzuheben, um die Qualität flächendeckend zu verbessern. Prähabilitationsprogramme mit Ausdauer-, Kraft- und intensivem Atemtraining und Ernährungstherapie verbessern das Patientenoutcome. Die aktuelle Therapie des Ösophaguskarzinoms erfolgt stadienabhängig und umfasst die endoskopische Resektion bei (sub-)mukosalen Low-risk-Tumoren (T1m1-3 bzw. T1sm1 "low risk"), die primäre Ösophagektomie bei submukosalen High-risk-Tumoren (T1a),
Submukosakarzinomen (T1sm2-3) und T2N0-Tumoren, die multimodale Therapie mittels neoadjuvanter Radiochemotherapie bzw. perioperativer Chemotherapie und Operation bei fortgeschrittenen Befunden. Die Ösophagektomie wird gegenwärtig einzeitig als sog. Hybridverfahren (Laparoskopie und muskelschonende Thorakotomie) oder als total minimal-invasive Operation (Laparoskopie und Thorakoskopie) durchgeführt.

Schlüsselwörter

Ösophaguskarzinom - Epidemiologie ·

Endoskopie $\cdot$ Mindestmenge $\cdot$ Prähabilitationsprogramme

\section{Surgical treatment of esophageal cancer-Indicators for quality in diagnostics and treatment}

\section{Abstract}

Background. Within the framework of the quality initiative of the German Society for General and Visceral Surgery (DGAV) a review article was compiled based on a systematic literature search. Recommendations for the current diagnostics and treatment of esophageal cancer were also elaborated. Methods. The systematic literature search was carried out in March 2019 according to the PRISMA criteria using the MEDLINE databank. The recommendations were formulated based on a consensus in the DGAV.

Results and conclusion. Operations below the currently valid minimum quantity threshold should no longer be carried out. There are many indications that the minimum quantity in Germany should be raised to $\geq 20$ resections/year/hospital in order to comprehensively improve the quality. Prehabilitation programs with endurance, strength and intensive breathing training as well as nutritional therapy improve patient outcome. The current treatment of esophageal cancer is stage-dependent and incorporates endoscopic resection of (sub)mucosal low-risk tumors (T1 $1 \mathrm{~m} 1-3$ or $\mathrm{T} 1 \mathrm{sm} 1$ low risk), primary esophagectomy of submucosal high-risk tumors (T1a), submucosal cancer (T1sm2-3) and T2N0 tumors, multimodal treatment with neoadjuvant chemoradiotherapy or perioperative chemotherapy and operations for advanced stages. Esophagectomy is nowadays carried out in one stage as a socalled hybrid procedure (laparoscopy and muscle-preserving thoracotomy) or as a total minimally invasive operation (laparoscopy and thoracoscopy)

\section{Keywords}

Esophageal cancer · Epidemiology · Endoscopy - Minimum number - Prehabilitation program
Submukosale Läsionen stellen den Untersucher vor besondere Probleme bezüglich der Dignität der Läsion. Durch besondere Tiefenbiopsietechniken („deep biopsy“, „bite-on-bite biopsy") kann die Erfolgsrate der Biopsie gesteigert werden bei gleichzeitig guter Blutungskontrolle und niedrigem Perforationsrisiko $[9,10]$.

\section{Computertomographie}

Nach der histologischen Sicherung eines Karzinoms sollte sich eine CT von Thorax, Abdomen und ggf. des Halses (bei hochsitzenden Tumoren) mit oralem und intravenösem Kontrastmittel anschließen. Das CT ermöglicht eine relativ gute Aussage über eine Metastasierung in parenchymatösen Organen wie Leber und Lunge, über die Position des Tumors, über eine mögliche Organüberschreitung und einen Einbruch in $\mathrm{Ge}$ - fäße oder Nachbarorgane. Eine Aussage bezüglich einer Lymphknotenmetastasierung ist nur eingeschränkt möglich, da die Größe von Lymphknoten nicht immer mit der Dignität korreliert [1].

\section{Endoskopischer Ultraschall}

Beim Nachweis von Fernmetastasen kann auf eine EUS-Untersuchung verzichtet werden. Sofern jedoch keine Fernmetastasen vorliegen, sollte zum Staging eine EUS-Untersuchung durch- 
geführt werden. Der EUS hat den Vorteil einer sehr hohen lokalen Ortsauflösung und bietet daher die höchste Treffsicherheit aller Verfahren zur Beurteilung der lokalen Infiltrationstiefe (T-Kategorie) und zur Beurteilung von Metastasen in regionäre Lymphknoten $[11,12]$. Das Verfahren erreicht bei der Beurteilung der T-Kategorie (für höhere T-Stadien) eine Sensitivität von $92 \%$ und eine Spezifität von 99\%. In Kombination mit einer Feinnadelbiospie können auch regionäre Lymphknoten abgeklärt werden und die Sensitivität und Spezifität des Verfahrens in der Beurteilung der Lymphknoten auf bis zu $97 \%$ bzw. $96 \%$ gesteigert werden $[11,12]$.

\section{PET-CT}

Zum Einsatz einer Positronenemissionstomographie(PET)-CT beim Primärstaging existieren mehrere Studien, die zeigen konnten, dass bei ca. 16-32\% der Patienten weitere Lymphknoten- oder Fernmetastasen diagnostiziert werden können, die durch EUS und CT nicht gefunden wurden [13-16]. Die Sensitivität und Spezifität der PET-CT wird dabei von einigen Autoren mit bis zu $91 \%$ bzw. $94 \%$ angegeben [15].

\section{UICC-TNM-System}

Entsprechend der deutschen Leitlinie sollte der histopathologische Befund der Operationsresektate die Größe, den histologischen Typ und die Art der neoplastischen Läsion mit Lokalisation in Bezug zum gastroösophagealen Übergang beschreiben. Es sollte eine Einteilung ins aktuelle UICC(Union for International Cancer Control)-TNMSystem erfolgen. Wichtig sind hierbei Grading, Lymphgefäß- und Veneninfiltration, Resektionsränder oral, aboral und zirkumferenziell sowie der Lymphknotenstatus. Sofern eine neoadjuvante Therapie erfolgte soll eine Regressionsgrading erfolgen.

\section{Response auf die neoadjuvante Therapie}

Derzeit existiert keine Untersuchung, die die Ansprechrate auf die neoadjuvante
Therapie ausreichend valide abschätzen kann [17]. Daher ist aktuell nach neoadjuvanter Therapie die Operation mit anschließender histopathologischer Aufarbeitung des Resektates weiterhin der Goldstandard einer kurativen Therapie. Im Rahmen des Restagings sollten Kontraindikationen für die Operation und das Auftreten von Fernmetastasen ausgeschlossen werden. Hierzu erscheinen eine erneute ÖGD und ein CT von Thorax und Abdomen ausreichend.

\section{Stadienabhängige kurative Therapie und Operations- indikation}

Die kurative Therapie des Ösophaguskarzinom variiert in Abhängigkeit vom jeweiligen Tumorstadium: Bei Frühkarzinomen (pT1) ist eine lokale Therapie mittels endoskopischer Mukosaresektion (EMR) bzw. Submukosadissektion (ESD) vertretbar, sofern keine Kontraindikationen wie Submukosainfiltration $>500 \mu \mathrm{m}$, lymphovaskuläre Infiltration (L1, V1) oder G3/4-Differenzierung vorliegen [1]. Nichtsdestotrotz ist ein nodales Metastasierungsrisiko von bis zu $34 \%$ bei zunehmender Infiltrationstiefe von pT1-Befunden mit Beteiligung der tieferen Schichten der Submukosa (sm2-3) zu beachten ( $\bullet$ Tab. 1; [18, 19]). Daher ist in solchen Fällen die Ösophagektomie mit regionärer Lymphadenektomie $\mathrm{zu}$ empfehlen. In einer retrospektiven Untersuchung zeigten sich keine Unterschiede im Überleben zwischen solchen Patienten mit primärer Operation bei Frühkarzinom im Vergleich zur operativen Resektion nach endoskopischer Intervention. Jedoch war die Rezidivrate nach alleiniger endoskopischer Resektion signifikant höher [20].

Analog hierzu sollten T2-Ösophagustumoren ohne Hinweis auf lokoregionäre Metastasierung direkt der Operation zugeführt werden oder aber alternativ wie bei T2-Tumoren mit lokalem Lymphknotenbefall bzw. T3- und T4a-Tumoren die multimodale Therapie mit perioperativer Chemotherapie bzw. neoadjuvanter Strahlenchemotherapie indiziert werden (• Tab. 1; [21-24]).

Die operative Resektion eines Ösophaguskarzinoms stellt einen der kom- plexesten viszeralchirurgischen Eingriffe dar und sollte dementsprechend an einem Zentrum mit entsprechender Expertise erfolgen, da hierdurch die postoperative Mortalität deutlich gesenkt werden kann [25]. Durch entsprechende Spezialisierung liegen die Raten der 30-Tageund 90-Tage-Mortalität bei 2,4\% bzw. $4,5 \%$ [26].

Zudem ist hier die standardisierte Erfassung möglicher Komplikationen gemäß der sog. Esophagectomy Complications Consensus Group (ECCG) gewährleistet [27]. Dabei werden typische intra-/postoperative Komplikationen wie Anastomoseninsuffizienzen, Interponatnekrosen, Lymphfisteln, Affektionen des N. laryneus recurrens, Vorhoffflimmern oder Pneumonie systematisch erfasst und gemäß der Clavien-Dindo-Klassifikation kategorisiert [27, 28]. Anastomoseninsuffizienzen werden auch in Zentren im Durchschnitt bei 11,4\% der Patienten beobachtet [26].

\section{Rekonstruktionstechniken}

Im Laufe der Zeit haben sich verschiedene Rekonstruktionstechniken etabliert: In Abhängigkeit von der Lage der Anastomose differenziert man zwischen hoch-thorakaler (Ivor Lewis; 56,3\%) und zervikaler (McKeown-)Ösophagektomie $(43,7 \%$; $[26,29])$. Wurde vormals der Großteil der Eingriffe offenchirurgisch durchgeführt, wird heutzutage nahezu die Hälfte der Eingriffe in minimal-invasiver Operationstechnik (MIE) vorgenommen [26]. Auch gibt es mittlerweile Kombinationsverfahren wie die Hybridösophagektomie, bei der die abdominelle Gastrolyse und Lymphadenektomie laparoskopisch erfolgt und die thorakale Resektion/Rekonstruktion offen-chirurgisch [30-33].

Die Datenlage hinsichtlich der Überlegenheit eines der genannten Verfahren ist uneinheitlich: Beide Rekonstruktionen (thorakal vs. zervikal) scheinen die gleiche klinische Sicherheit für den Patienten zu bieten, jedoch ergaben Metaanalysen, dass die Ivor-Lewis-Ösophagektomie hinsichtlich des Krankenhausaufenthaltes und Blutverlustes möglicherweise überlegen ist $[34,35]$. Systematische, prospektive Daten stehen noch aus. 
Tab. 1 Evidenzunterlegte Qualitätsindikatoren für die Diagnostik und die chirurgische Therapie beim Ösophaguskarzinom

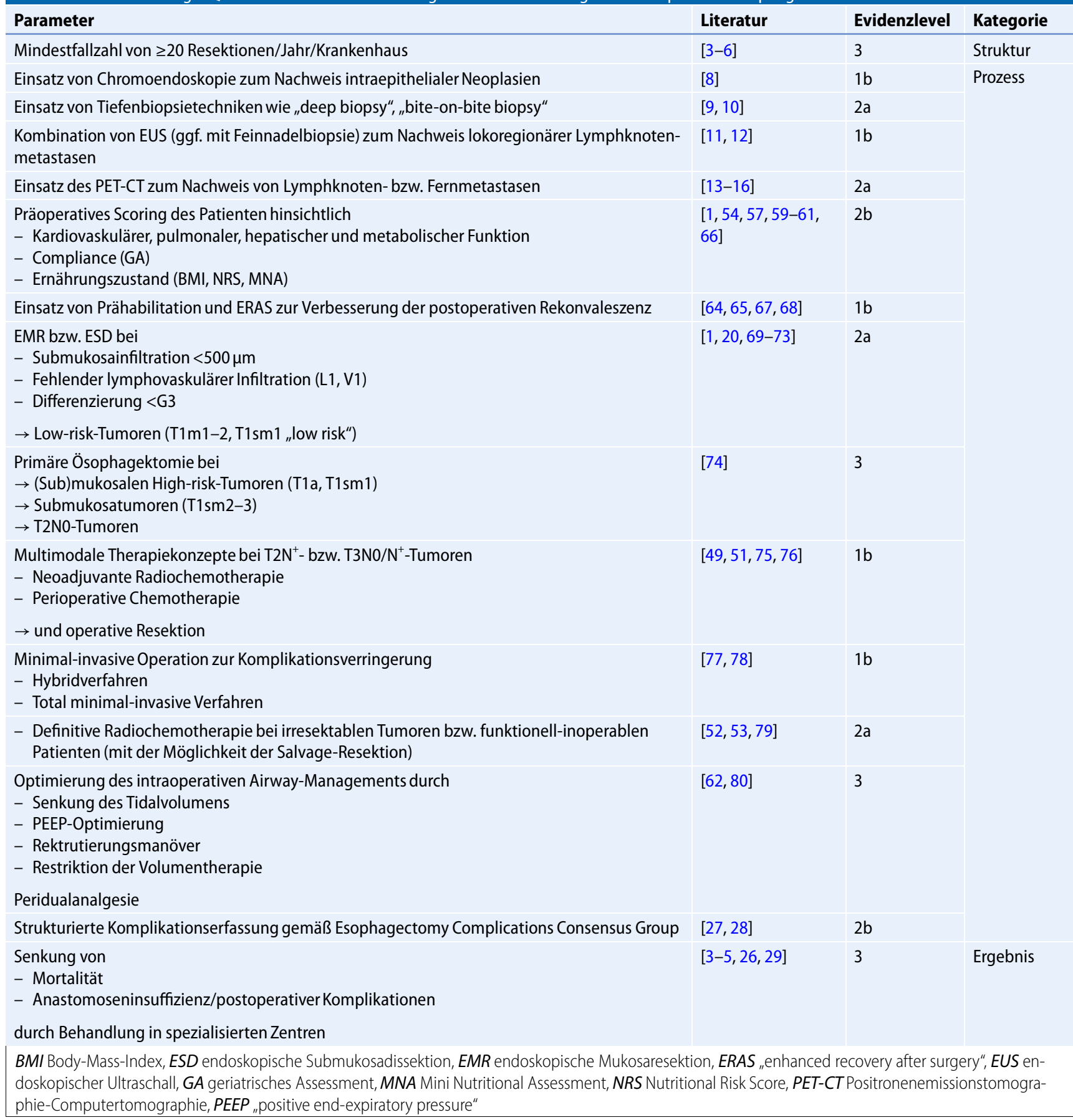

Bezüglich des Einsatzes minimal-invasiver Techniken konnte für die Hybridösophagektomie eine signifikante Überlegenheit in den Ergebnissen in der postoperativen Morbidität bei gleichwertigen onkologischen Ergebnissen prospektiv-randomisiert bewiesen werden [28]. Die robotisch-assistierte Ösophagektomie stellt die jüngste Weiterentwicklung hinsichtlich der minimal-invasiven Operationsmethodik dar [36-39].

\section{Lymphadenektomie}

Im Rahmen der Ösophagektomie erfolgt die 2-Feld-Lymphadenektomie des Abdomens und unteren Mediastinums mit der Resektion von mindestens 20 bis 30 Lymphknoten. Mittlerweile ist hier jedoch davon auszugehen, dass nicht die Gesamtzahl der resezierten Lymphknoten von prognostischer Relevanz ist, sondern vielmehr das Verhältnis tumorpositiver $\mathrm{zu}$ negativen Lymphknoten („lymph node ratio“; [40]). 


\begin{tabular}{|l}
\hline Infobox 1 Operative Therapie \\
\hline - Die Ösophagektomie wird heutzuta- \\
ge einzeitig als sog. Hybridverfahren \\
(Laparoskopie und muskelschonende \\
Thorakotomie) oder als total minimal-in- \\
vasive Operation (Laparoskopie und \\
Thorakoskopie) durchgeführt. \\
- Rekonstruktives Grundprinzip ist die \\
Wiederherstellung der Passage im oberen \\
Gastrointestinaltrakt mit Bildung eines \\
Schlauchmagens und Ösophagogastro- \\
stomie. \\
Seltener erfolgen Dünn und Dickdarmin- \\
terpositionen zur Passagerekonstruktion \\
(Ösophagojejunostomie bzw. Ösophago- \\
kolostomie). \\
In Abhängigkeit von der Lage der \\
Anastomose unterscheidet man zwischen \\
der Ösophagektomie nach Ivor-Lewis \\
(intrathorakale Anastomose) und der \\
Resektion nach McKoewn (zervikale \\
Anastomose). \\
Im Rahmen der Ösophagektomie erfolgt \\
die 2-Feld-Lymphadenektomie des \\
Abdomens und unteren Mediastinums \\
mit der Resektion von mindestens 20 bis \\
30 Lymphknoten. \\
- Bei gastroösophagealen Übergangstu- \\
moren vom Typ AEG II ist alternativ die \\
Resektion mittels transhiatal erweiterter \\
Gastrektomie und Rekonstruktion mittels \\
Ösophagojejunostomie eine Option. \\
\hline
\end{tabular}

\section{Tumoren des ösophagogastralen Übergangs}

Eine besondere Herausforderung stellt die Resektion der Tumoren des ösophagogastralen Übergangs (Adenokarzinome des ösophagogastralen Übergangs, AEG) dar. AEG-II-Tumoren können neben der Ösophagektomie auch mittels transhiatal erweiterter Gastrektomie (TEG) versorgt werden. Hinweise aus Beobachtungsstudien, ob der rein abdominelle Zugang der TEG zu weniger pulmonalen bzw. funktionellen Komplikationen führt und/oder mit einer höheren Lebensqualität bei vergleichbaren onkologischen Ergebnissen verbunden ist, werden aktuell in der internationalen CARDIA-Studie prospektiv randomisiert überprüft werden [41-43].

In - Infobox 1 sind die operativen Strategien beim Ösophaguskarzinom zusammengefasst.

\section{Perioperative multimodale onkologische Therapiekonzepte}

Die perioperative Chemotherapie und die neoadjuvante Radiochemotherapie verbessern das Langzeitüberleben beim nichtmetastasierten Ösophaguskarzinom in den Stadien T2-T4 NX M0 im Vergleich zur alleinigen chirurgischen Resektion signifikant ([44-46]; • Tab. 2). Die Operation wird in den multimodalen Therapieschemata 3 bis 8 Wochen nach Ende der neoadjuvanten Therapie durchgeführt und beeinflusst die postoperative Morbidität und Mortalität im Vergleich zur alleinigen Operation nicht [44-49]. Die postoperative Mortalität in den aktuellen multimodalen Protokollen beträgt 4-8\% [44-46, 49]. Für ein verlängertes Intervall zwischen dem Ende der neoadjuvanten Behandlung und dem Zeitpunkt der Operation gibt es aktuell keine überzeugende Evidenz [50]. Beim Plattenepithelkarzinom der Speiseröhre wird durch den aktuellen internationalen Standard mit einer Radiochemotherapie (41,4 Gy plus Carboplatin/Paclitaxel) das mediane Gesamtüberleben auf 82 Monate gegenüber 21 Monaten (Hazard Ratio [HR] 0,48) bei der alleinigen chirurgischen Therapie gesteigert [51]. Beim Adenokarzinom der Speiseröhre verbessert die neoadjuvante Radiochemotherapie das mediane Gesamtüberleben auf 43 Monate gegenüber 27 Monaten (HR 0,73; [51]). Im Gegensatz zum Plattenepithelkarzinom wird beim Adenokarzinom das Langzeitüberleben auch durch die perioperative Chemotherapie verbessert. Im Vergleich zur alleinigen Operation zeigen die prospektiv-randomisierten Studien MAGIC (Epirubricin, Cisplatin, 5-FU) und FFCD9703 (Cisplatin, 5-FU) signifikante Überlebensvorteile mit 5-JahresÜberlebensraten von 36 vs. $23 \%$ (HR 0,75 ) bzw. 38 vs. $24 \%$ (HR 0,69; [44, 45]). Der aktuelle chemotherapeutische Standard beim Adenokarzinom wird durch die perioperative FLOT-Therapie (5-Fluoruracil, Leukovorin, Oxaliplatin, Docetaxel) mit einem medianen Gesamtüberleben von 50 Monaten gebildet [49]. Welchem der beiden genannten multimodalen Therapiekonzepte im Hinblick auf Kurz- und Langzeitergebnisse beim
Adenokarzinom der Vorzug zu geben ist, ist ungeklärt und international umstritten. Die Frage ist Gegenstand der in Deutschland aktuell laufenden multizentrischen ESOPEC-Studie [21].

Bei intendiert definitiv radiochemotherapeutisch behandelten Ösophaguskarzinomen mit persistierendem Tumor oder lokalem Tumorrezidiv kann eine Salvage-Resektion und damit ebenfalls ein multimodales Therapiekonzept indiziert sein. Die zu diesem Konzept vorliegenden Daten zeigen eine höhere Morbidität und Mortalität ( 9,5 vs. $4,5 \%$ ) bei der Salvage-Resektion im Vergleich zur geplanten postneoadjuvanten Resektion [52]. Die vorliegenden $\mathrm{Da}$ ten zum Langzeitoutcome des SalvageOperationskonzepts sind unzureichend, weisen aber auf zu mindestens gleichwertige Überlebenszeiten im Vergleich zur geplanten Operation hin [53].

\section{Perioperatives Management}

\section{Risikoevaluation und -stratifizierung}

Eine differenzierte Risikoevaluation und -stratifizierung, möglichst vor Beginn einer neoadjuvanten Therapie, ist essenziell für ein erfolgreiches Outcome nach onkologischer Ösophagektomie. Hierzu haben sich, neben einer umfassenden Abklärung der kardiovaskulären, pulmonalen, hepatischen und metabolischen Funktion [1], validierte Scoringsysteme etabliert mit Korrelation zwischen präoperativem Status und postoperativer Morbidität und Letalität. Dabei ist O-POSSUM derzeit der am besten validierte, international angewandte klinische Risikoscore, der zur Prädiktion der postoperativen Morbidität und Mortalität nach Ösophagektomie zur Verfügung steht [54].

Neben dem Risikoscoring ist die Einschätzung der Compliance des Patienten für den postoperativen Verlauf entscheidend. Seine aktive Mitarbeit ist essenziell, um insbesondere die pulmonale Morbidität durch intensives Atemtraining zu vermindern.

Ältere Patienten haben bekanntermaßen ein größeres Risiko für postoperative Komplikationen nach Ösophagektomie 
Tab. 2 Nichtmetastasiertes Ösophaguskarzinom. Stadienadaptierte Auswahl des Therapieschemas

\begin{tabular}{|c|c|c|}
\hline Tumorstadium & TNM-Stadium & Behandlung \\
\hline $\begin{array}{l}\text { Mukosa- } \\
\text { karzinome }\end{array}$ & $\begin{array}{l}\text { cT1a cN- } \\
\text { "low risk" }\end{array}$ & \multirow[t]{2}{*}{ Endoskopische Resektion } \\
\hline $\begin{array}{l}\text { Submukosa- } \\
\text { karzinome }\end{array}$ & $\begin{array}{l}\text { cT1b sm } 1 \mathrm{cN}^{-} \\
\text {"low risk" }\end{array}$ & \\
\hline $\begin{array}{l}\text { Mukosa- } \\
\text { karzinome }\end{array}$ & $\begin{array}{l}\text { cT1a cN- } \\
\text { "high risk" }\end{array}$ & \multirow[t]{4}{*}{$\begin{array}{l}\text { Thorakoabdominelle } \\
\text { Ösophagusresektion + 2-Feld-Lymphadenektomie }\end{array}$} \\
\hline $\begin{array}{l}\text { Submukosa- } \\
\text { karzinome }\end{array}$ & cT1b sm $2 / 3 \mathrm{cN}^{-}$ & \\
\hline \multirow{2}{*}{$\begin{array}{l}\text { Lokalisierte } \\
\text { Karzinome }\end{array}$} & $\mathrm{cT} 1 \mathrm{cN}^{+}$ & \\
\hline & $\mathrm{CT} 2 \mathrm{cN}^{-}$ & \\
\hline \multirow{3}{*}{$\begin{array}{l}\text { Lokal fortge- } \\
\text { schrittene } \\
\text { Karzinome }\end{array}$} & $\mathrm{cT} 1-2 \mathrm{CN}^{+}$ & \multirow{3}{*}{$\begin{array}{l}\text { Thorakoabdominelle } \\
\text { Ösophagusresektion + 2-Feld-Lymphadenektomie plus } \\
\text { AC/PEC: neoadjuvante Radiochemotherapie (z. B. 41,4 Gy, } \\
\text { Carboplatin + Paclitaxel) oder AC: perioperative } \\
\text { Chemotherapie 3-Fach-Kombination (Docetaxel, } \\
\text { Platin + Flouropyrimidin) }\end{array}$} \\
\hline & $\mathrm{CT} 3 \mathrm{CN}^{-/+}$ & \\
\hline & cT4a cN ${ }^{-1+}$ & \\
\hline
\end{tabular}

[55]. Hier wird zusätzlich ein prätherapeutisches geriatrisches Assessment (GA) empfohlen, um ggf. eine risikoadaptierte Therapiemodifikation (z.B. der neoadjuvanten Therapie) durchzuführen [56] - basierend auf der individuellen „frailty“ (Gebrechlichkeit) bzw. „prefrailty" sowie der Funktions- und Fähigkeitseinschränkungen im GA (gemäß den Empfehlungen der AG „Geriatrische Onkologie“ der Deutschen Gesellschaft für Hämatologie und Medizinische Onkologie [DGHO]).

Pragmatisch und zeiteffizient durchzuführen sind präoperativ der 6-minutewalk-Test (6 MWT; [57]) sowie der Shuttle-walk-Test (SWT; [58]). Ein weiteres einfaches und genaues Testverfahren zur exakten Beurteilung der Leistungsfähigkeit stellt die Spiroergometrie dar. Hochrisikopatienten sollten mittels Ergometrie identifiziert werden [59]. Die präoperative Leistungsfähigkeit stellt einen wichtigen Prädiktor der postoperativen Prognose bei Patienten mit Ösophaguskarzinom dar $[58,60]$.

\section{Ernährungsstatus}

Ein prätherapeutisches Screening beim Ösophaguskarzinom muss hinsichtlich Malnutrition und Katabolie basierend auf dem vorhandenen Ausmaß der Dysphagie erfolgen [1]. Die Erfassung des
BMI (Body-Mass-Index), des NRS (Nuni Nutritional Assessment) und einer potenziellen Sarkopenie impliziert die Applikation oraler Trinklösungen, supportiver enteraler Nahrungssubstanzen bzw. evt. einer i.v. Supplementierung.

Risikopatienten mit einem Gewichtsverlust von $>10 \%$ in den vorausgegangenen 3 Monaten, einem $\mathrm{BMI}<18,5 \mathrm{~kg} / \mathrm{m}^{2}$ oder einer Serumalbuminkonzentration $<30 \mathrm{~g} / \mathrm{l}$ sollten präoperativ mithilfe einer Ernährungstherapie vorbereitet werden [61].

\section{Optimiertes anästhesiologisches Management}

Die Beatmung mit niedrigem Tidalvolumen, Optimierung des PEEP („positive end-expiratory pressure") sowie die routinemäßige Anwendung von Rekrutierungsmanövern reduzieren postoperative pulmonale Komplikationen. Die Flüssigkeitstherapie sollte eine Überwässerung vermeiden, welche ebenso einen bekannten Risikofaktor für pulmonale Komplikationen sowie Anastomoseninsuffizienzen darstellt. Die thorakale Periduralanalgesie reduziert die systemische inflammatorische Reaktion, vermindert pulmonale Komplikationen und verbessert die postoperative Schmerzkontrolle [62]. tritional Risk Score), des MNA (Mi-
Prähabilitation und „enhanced recovery after surgery"

Die funktionelle Rekonvaleszenz ist entscheidend für das postoperative Ergebnis. Dabei bedingt in der Ösophaguschirurgie die Prähabilitation zur Optimierung der funktionellen Reserven vor der Operation, z.B. im Rahmen der neoadjuvanten Therapie, eine beschleunigte Erholungsphase und eine Reduktion von Komplikationen. Zu den multimodalen Präkonditionierungskonzepten zählen eine Kombination aus Ausdauer-, Kraftund intensivem Atemtraining sowie einer Ernährungstherapie unter Berücksichtigung des individuellen präoperativen Leistungsniveaus und des Ernährungsstatus, idealerweise unter professionellem Monitoring.

„Enhanced-recovery-after-surgery“ (ERAS)-Programme fokussieren gezielt die Steuerung einer standardisierten Behandlung nach der Operation mit dem Ziel der Minimierung postoperativer Komplikationen und der Beschleunigung der Rekonvaleszenz [63]. Während für den positiven Effekt der Prähabilitation vor der Ösophaguschirurgie evidenzbasierte Daten in der Literatur vorliegen $[64,65]$, sind die ERAS-Pathways zwar vielversprechend, um einen weiteren Benefit zu erzielen, wenngleich zum aktuellen Zeitpunkt große, prospektive Multicenterstudien mit entsprechender Evidenz fehlen.

\section{Fazit für die Praxis}

- Es spricht vieles dafür, die Mindestmenge in Deutschland auf $\geq 20$ Resektionen/Jahr/Krankenhaus anzuheben, um die Qualität (Senkung der Morbidität bzw. Mortalität) flächendeckend zu verbessern.

- Für die Diagnostik des Ösophaguskarzinoms sollte neben der Anamnese und der körperlichen Untersuchung die Durchführung einer Ösophagogastroduodenoskopie (ÖGD) mit Biposie, die endoskopische Sonographie (EUS) und die Computertomographie (CT) des Thorax/ Abdomens durchgeführt werden.

- Die Therapie des Ösophaguskarzinoms erfolgt stadienabhängig und 
umfasst die endoskopische Resektion bei (sub-)mukosalen Low-riskTumoren (T1m1-3 bzw. T1sm1 low risk), die primäre Ösophagektomie bei submukosalen High-risk-Tumoren (T1a), Submukosakarzinomen (T1sm2-3) und T2N0-Tumoren, die multimodale Therapie mittels neoadjuvanter Radiochemotherapie bzw. perioperativer Chemotherapie und Operation bei fortgeschrittenen Befunden.

- Die intra-/postoperative Komplikationen wie Anastomoseninsuffizienzen, Interponatnekrosen, Lymphfisteln, Affektionen des N. laryneus recurrens, Vorhoffflimmern oder Pneumonie sollten gemäß der sog. Esophagectomy Complications Consensus Group (ECCG) standardisiert erfasst werden.

- Zur präoperativen Risikoabschätzung gehört die Untersuchung der kardiovaskulären, pulmonalen, hepatischen und metabolischen Funktion. Zudem sollte der Ernährungszustand des Patienten mittels BMI (Body Mass Index), NRS (Nutritional Risk Score) oder MNA (Mini Nutritional Assessment) erfasst und eine Malnutrition ggf. bereits präoperativ behandelt werden.

- Prähabilitationsprogramme mit Ausdauer-, Kraft- und intensivem Atemtraining sowie einer Ernährungstherapie verbessern das Patientenoutcome deutlich.

- Intraoperativ profitiert der Patient von einer restriktiven Volumentherapie, niedrigem Tidalvolumen, Optimierung des PEEP (,positive end-expiratory pressure") sowie des Einsatzes der Periduralanalgesie.

\section{Korrespondenzadresse}

Prof. Dr. med. Jens Hoeppner Klinik für Allgemein- und Viszeralchirurgie, Universitätsklinikum Freiburg, Medizinische Fakultät, Albert-Ludwigs-Universität Freiburg im Breisgau

Hugstetter Straße 55, 79106 Freiburg, Deutschland jens.hoeppner@uniklinik-freiburg.de DEAL.
Einhaltung ethischer Richtlinien

Interessenkonflikt. J. Hoeppner, P.S. Plum, H. Buhr, I. Gockel, D. Lorenz, M. Ghadimi und C. Bruns geben an, dass kein Interessenkonflikt besteht.

Für diesen Beitrag wurden von den Autoren keine Studien an Menschen oder Tieren durchgeführt. Für die aufgeführten Studien gelten die jeweils dort angegebenen ethischen Richtlinien.

Open Access Dieser Artikel wird unter der Creative Commons Namensnennung 4.0 International Lizenz veröffentlicht, welche die Nutzung, Vervielfältigung, Bearbeitung, Verbreitung und Wiedergabe in jeglichem Medium und Format erlaubt, sofern Sie den/die ursprünglichen Autor(en) und die Quelle ordnungsgemäßnennen, einen Link zur Creative Commons Lizenz beifügen und angeben, ob Änderungen vorgenommen wurden.

Die in diesem Artikel enthaltenen Bilder und sonstiges Drittmaterial unterliegen ebenfalls der genannten Creative Commons Lizenz, sofern sich aus der Abbildungslegende nichts anderes ergibt. Sofern das betreffende Material nicht unter der genannten Creative Commons Lizenz steht und die betreffende Handlung nicht nach gesetzlichen Vorschriften erlaubt ist, ist für die oben aufgeführten Weiterverwendungen des Materials die Einwilligung des jeweiligen Rechteinhabers einzuholen.

Weitere Details zur Lizenz entnehmen Sie bitte der Lizenzinformation auf http://creativecommons.org/ licenses/by/4.0/deed.de.

\section{Literatur}

1. Porschen R, Fischbach W, Gockel I, Hollerbach S, Hölscher A, Jansen PL et al (2019) S3-Leitlinie Diagnostik und Therapie der Plattenepithelkarzinome und Adenokarzinome des ösophagus. Z Gastroenterol 57(3):336-418 (http://www.ncbi. nlm.nih.gov/pubmed/30861559)

2. Moher D, Liberati A, Tetzlaff J, Altman DG, PRISMA Group (2009) Preferred reporting items for systematic reviews and meta-analyses: the PRISMA statement. PLoS Med 6(7):e1000097-21 (http:// www.ncbi.nlm.nih.gov/pubmed/19621072)

3. Busweiler LAD, Wijnhoven BPL, van Berge Henegouwen MI, Henneman D, van Grieken NCT, Wouters MWJM et al (2016) Early outcomes from the Dutch Upper Gastrointestinal Cancer Audit. Br J Surg 103(13):1855-1863 (http://www.ncbi.nIm. nih.gov/pubmed/27704530)

4. Kjaer DW, Larsson $H$, Svendsen LB, Jensen LS (2017) Changes in treatment and outcome of oesophageal cancer in Denmark between 2004 and 2013. Br J Surg 104(10):1338-1345 (http:// www.ncbi.nlm.nih.gov/pubmed/28718940)

5. Nimptsch U, Peschke D, Mansky T (2017) Mindestmengen und Krankenhaussterblichkeit Beobachtungsstudie mit deutschlandweiten Krankenhausabrechnungsdaten von 2006 bis 2013. Gesundheitswesen 79(10):823-834 (http:// www.ncbi.nlm.nih.gov/pubmed/27050140)

6. Nimptsch U, Haist T, Krautz C, Grützmann R, Mansky T, Lorenz D (2018) Fallzahl, Krankenhaussterblichkeit und Komplikationsmanagement in der ösophaguschirurgie. Dtsch Arztebl Int
115(47):793-800 (http://www.ncbi.nlm.nih.gov/ pubmed/30636674)

7. Behrens A, Labenz J, Schuler A, Schröder W, RünziM,Steinmann RU etal (2013) Wie sicherist die Sedierung in der gastrointestinalen Endoskopie? Eine multizentrische Auswertung von 388404 Endoskopien und Auswertung der Daten aus prospektiv geführten Komplikationsregistern von Mitgliedern der Arbeitsgemeinschaft leitender Gastroent. Z Gastroenterol 51(5):432-436 (http:// www.ncbi.nlm.nih.gov/pubmed/23681895)

8. Qumseya BJ, Wang H, Badie N, Uzomba RN, Parasa S, White DL et al (2013) Advanced imaging technologies increase detection of dysplasia and neoplasia in patients with barrett's esophagus: a meta-analysis and systematic review. Clin Gastroenterol Hepatol 11:1562-1570.e2 (http:// www.ncbi.nlm.nih.gov/pubmed/23851020)

9. Tae HJ, Lee HL, Lee KN, Jun DW, Lee OY, Han DS et al (2014) Deep biopsy via endoscopic submucosal dissection in upper gastrointestinal subepithelial tumors: a prospective study. Endoscopy 46(10):845-850 (http://www.ncbi.nlm.nih.gov/ pubmed/25111136)

10. Ji JS, Lee BI, Choi KY, Kim BW, Choi H, Huh M et al (2009) Diagnostic yield of tissue sampling using a bite-on-bite technique for incidental subepithelial lesions. Korean J Intern Med 24(2):101-105 (http://www.ncbi.nlm.nih.gov/ pubmed/19543487)

11. Thosani N, Singh H, Kapadia A, Ochi N, Lee JH Ajani J et al (2012) Diagnostic accuracy of EUS in differentiating mucosal versus submucosal invasion of superficial esophageal cancers: a systematic review and meta-analysis. Gastrointest Endosc 75(2):242-253 (http://www.ncbi.nlm.nih. gov/pubmed/22115605)

12. Puli SR, Reddy JBK, Bechtold ML, Antillon D, Ibdah JA, Antillon MR (2008) Staging accuracy of esophageal cancer by endoscopic ultrasound: a meta-analysis and systematic review. World J Gastroenterol 14:1479-1490 (http://www.ncbi. nlm.nih.gov/pubmed/18330935)

13. Flamen $P$, Lerut $A$, Van Cutsem $E$, De Wever $W_{t}$ Peeters M, Stroobants S et al (2000) Utility of positron emission tomography for the staging of patients with potentially operable esophageal carcinoma. JClin Oncol 18(18):3202-3210 (http:// www.ncbi.nlm.nih.gov/pubmed/10986052)

14. Heeren PAM, Jager PL, Bongaerts F, Van Dullemen H, Sluiter W, Plukker JTM (2004) Detection of distant metastases in esophageal cancer with $18 \mathrm{~F}$ FDG PET. J Nucl Med 45(6):980-987 (http://www. ncbi.nlm.nih.gov/pubmed/15181133)

15. Noble F, Bailey D, SWCIS Upper Gastrointestinal Tumour Panel, Tung K, Byrne JP (2009) Impact of integrated PET/CT in the staging of oesophageal cancer-a UK population-based cohort study. Clin Radiol 64(7):699-705 (http://www.ncbi.nlm.nih. gov/pubmed/19520214)

16. Barber TW, Duong CP, Leong T, Bressel $M$, Drummond EG, Hicks RJ (2012) 18F-FDG PET/CT has a high impact on patient management and provides powerful prognostic stratification in the primary staging of esophageal cancer: A prospective study with mature survival data. J Nucl Med 53(6):864-871 (http://www.ncbi.nlm. nih.gov/pubmed/22582047)

17. Cosgrove ND, Mullady DK (2018) Endoscopic evaluation of the esophageal cancer patient after chemoradiotherapy for persistent/recurrent cancer. Vol. 31, Diseases of the Esophagus. http:// www.ncbi.nlm.nih.gov/pubmed/29931309. Zugegriffen: 1.Dez. 2019 
18. Hölscher AH, Bollschweiler E, Schröder W, Metzger R, Gutschow C, Drebber U (2011) Prognostic impact of upper, middle, and lower third mucosal or submucosal infiltration in early esophageal cancer. Ann Surg 254(5):802-808 (http://content. wkhealth.com/linkback/openurl?sid=WKPTLP: landingpage\&an=00000658-201111000-00018)

19. Lorenz D, Origer J, Pauthner M, Graupe F, FisselerEckhoff A, Stolte M et al (2014) Prognostic risk factors of early esophageal adenocarcinomas. Ann Surg 259(3):469-476 (http://www.ncbi.nlm.nih. gov/pubmed/24096754)

20. PlumPS, HölscherAH, PachecoGodoyK, SchmidtH, Berlth F, Chon SH et al (2018) Prognosis of patients with superficial T1 esophageal cancer who underwent endoscopic resection before esophagectomy-A propensity score-matched comparison. Surg Endosc 32(9):3972-3980. https://doi.org/10.1007/s00464-018-6139-7

21. Hoeppner J, LordickF, Brunner T, Glatz T, Bronsert $P_{\text {, }}$ Röthling $N$ et al (2016) ESOPEC: prospective randomized controlled multicenter phase III trial comparing perioperative chemotherapy (FLOT protocol) to neoadjuvant chemoradiation (CROSS protocol) in patients with adenocarcinoma of the esophagus (NCT02509286). BMC Cancer 16(1):503 (http://bmccancer.biomedcentral.com/articles/ 10.1186/s12885-016-2564-y)

22. Goense L, van der Sluis PC, van Rossum PSN, van de Horst S, Meijer GJ, Haj MN et al (2017) Perioperative chemotherapy versus neoadjuvant chemoradiotherapy for esophageal or GEJ adenocarcinoma: a propensity score-matched analysis comparing toxicity, pathologic outcome, and survival. J Surg Oncol 115(7):812-820. https://doi.org/10.1002/ jso. 24596

23. Porschen R, BuckA, FischbachW, Gockell, Görling U, Grenacher L et al (2015) S3-Guideline of diagnostic and therapy of squamous cell carcinoma and adenocarcinoma of the esophagus (Long Version 1.0-September 2015, AWMF-registry number: 021/023OL).ZGastroenterol 53(11):1288-1347

24. Borggreve AS, Kingma BF, Domrachev SA, Koshkin MA, Ruurda JP, van Hillegersberg $R$ et al (2018) Surgical treatment of esophageal cancer in the era of multimodality management. Ann N Y Acad Sci 1434:192-209

25. Hoag JR, Resio BJ, Monsalve AF, Chiu AS, Brown LB, Herrin J et al (2019) Differential Safety Between Top-Ranked Cancer Hospitals and Their Affiliates for Complex Cancer Surgery. JAMA Netw Open 2(4):e191912 (http://www.ncbi.nlm.nih. gov/pubmed/30977848)

26. Low DE, Kuppusamy MK, Alderson D, Cecconello I, Chang AC, Darling G et al (2019) Benchmarking complications associated with esophagectomy. Ann Surg 269(2):291-298

27. Low DE, Alderson D, Cecconello I, Chang AC, Darling GE, D'Journo XB et al (2015) International consensus on standardization of data collection for complications associated with esophagectomy: esophagectomy complications consensus group (ECCG). Ann Surg 262(2):286-294 (https://insights. ovid.com/pubmed?pmid $=25607756$ )

28. van der Werf LR, Busweiler LAD, van Sandick JW, van Berge Henegouwen MI, Wijnhoven BPL, Dutch Upper GI Cancer Audit (DUCA) group (2019) Reporting national outcomes after esophagectomy and gastrectomy according to the esophageal complications consensus group (ECCG). Ann Surg. https://doi.org/10.1097/SLA.0000000000003210

29. Schmidt HM, Gisbertz SS, Moons J, Rouvelas I, Kauppi J, Brown A et al (2017) Defining benchmarks for transthoracic esophagectomy: a multicenter analysis of total minimally invasive Eesophagectomy in low risk patients. Ann Surg 266(5):814-821 (http://www.ncbi.nlm.nih.gov/ pubmed/28796646)

30. Voron T, Lintis A, Piessen G (2019) Hybrid esophagectomy. J Thorac Dis 11(Suppl 5):S723-S727 (http://www.ncbi.nlm.nih.gov/ pubmed/31080650)

31. Mariette C, Markar SR, Dabakuyo-Yonli TS, Meunier B, Pezet D, Collet D et al (2019) Hybrid minimally invasive esophagectomy for esophageal cancer. NEngl J Med 380(2):152-162

32. Scarpa M, Cavallin F, Saadeh LM, Pinto E, Alfieri R, Cagol M et al (2016) Hybrid minimally invasive esophagectomy for cancer: impact on postoperative inflammatoryand nutritional status. Dis Esophagus 29(8):1064-1070

33. Allaix ME, Long JM, Patti MG (2016) Hybrid Ivor lewis Eesophagectomy for esophageal cancer. J Laparoendosc Adv Surg Tech 26(10):763-767

34. Deng J, Su Q, Ren Z, Wen J, Xue Z, Zhang L et al (2018) Comparison of short-term outcomes between minimally invasive McKeown and Ivor Lewisesophagectomy foresophageal or junctional cancer: a systematic review and meta-analysis. Onco Targets Ther 11:6057-6069. https://doi.org/ 10.2147/OTT.S169488

35. van Workum F, Berkelmans GH, Klarenbeek BR, Nieuwenhuijzen GAP, Luyer MDP, Rosman C (2017) McKeown or Ivor Lewis totally minimally invasive esophagectomy for cancer of the esophagus and gastroesophageal junction: Systematic review and meta-analysis. JThorac Dis 9(Suppl 8):S826-S833

36. Rolff HC, Ambrus RB, Belmouhand M, Achiam MP, Wegmann M, Siemsen M et al (2017) Robotassisted hybrid esophagectomy is associated with a shorter length of stay compared to conventiona transthoracic esophagectomy: a retrospective study. Minim Invasive Surg 2017:1-6

37. Jin D, Yao L, Yu J, Liu R, Guo T, Yang Ket al (2019) robotic-assisted minimally invasive esophagectomy versus the conventional minimally invasive one: a meta-analysis and systematic review. Int J Med Robot Comput Assist Surg 15(3):1-11

38. Zhang Y, Han Y, Gan Q, Xiang J, Jin R, Chen K et al (2019) Early outcomes of robot-assisted versus thoracoscopic-assisted Ivor lewis esophagectomy for esophageal cancer: a propensity scorematched study. Ann Surg Oncol 26(5):1284-1291. https://doi.org/10.1245/s10434-019-07273-3

39. van der Sluis $P C$, van der Horst $S$, May $A M$ Schippers C, Brosens LAA, Joore HCA et al (2019) Robot-assisted minimally invasive thoracolaparoscopic esophagectomy versus open transthoracic esophagectomy for resectable esophageal cancer: a randomized controlled trial. Ann Surg 269(4):621-630

40. Tsai TC, Miller J, Andolfi C, Whang B, Fisichella PM (2018) Surgical evaluation of lymph nodes in esophageal adenocarcinoma: Standardized approach or personalized medicine? Eur J Surg Oncol 44(8):1177-1180

41. Fuchs $H$, Hölscher $A H$, Leers J, Bludau $M$ Brinkmann S, Schröder W et al (2016) Long-term quality of life after surgery for adenocarcinoma of the esophagogastric junction: extended gastrectomy or transthoracic esophagectomy? Gastric Cancer 19(1):312-317

42. Blank S, Schmidt T, Heger P, Strowitzki MJ, Sisic L, Heger U et al (2018) Surgical strategies in true adenocarcinoma of the esophagogastric junction (AEG II): thoracoabdominal or abdominal approach? Gastric Cancer 21(2):303-314
43. Heger P, BlankS, Gooßen K, NienhüserH, DienerMK, Ulrich A et al (2019) Thoracoabdominal versus transhiatal surgical approaches for adenocarcinoma of the esophagogastric junction-a systematic review and meta-analysis. Langenbecks Arch Surg 404(1):103-113 (http://www.ncbi.nlm.nih.gov/ pubmed/30607534)

44. Ychou $M$, Boige V, Pignon JP, Conroy $T$, Bouché $O$, Lebreton $\mathrm{G}$ et al (2011) Perioperative chemotherapy compared with surgery alone for resectable gastroesophageal adenocarcinoma: an FNCLCC and FFCD multicenter phase III trial. J Clin Oncol 29(13):1715-1721 (http://www.ncbi.nlm.nih.gov/ pubmed/21444866)

45. Cunningham D, Allum WH, Stenning SP, Thompson JN, Van De Velde CJH, Nicolson M et al (2006) Perioperative chemotherapy versus surgery alone for resectable gastroesophageal cancer. N Engl J Med 355(1):11-20

46. Van Hagen $P$, Hulshof MCCM, Van Lanschot JJB, Steyerberg EW, Van Berge Henegouwen MI, Wijnhoven BPL et al (2012) Preoperative chemoradiotherapy for esophageal or junctional cancer. N Engl J Med 366(22):2074-2084 (http://www. ncbi.nlm.nih.gov/pubmed/22646630)

47. Burmeister B, Smithers M, Gebski V, Denham J Devitt $P, A c k l a n d ~ S$ et al (2005) Surgery alone versus chemoradiotherapy followed by surgery for resectable cancer of the oesophagus: A randomised controlled phase III trial. Lancet Oncol 6(9):659-668 (http://www.ncbi.nlm.nih. gov/pubmed/16129366)

48. Tepper J, Krasna MJ, Niedzwiecki D, Hollis D, Reed CE, Goldberg R et al (2008) Phase III trial of trimodality therapy with cisplatin, fluorouracil, radiotherapy, and surgery compared with surgery alone for esophageal cancer: CALGB 9781. J Clin Oncol 26(7):1086-1092 (http://www.ncbi.nlm. nih.gov/pubmed/18309943)

49. Al-Batran SE, Homann N, Pauligk C, Goetze TO Meiler J, Kasper S et al (2019) Perioperative chemotherapy with fluorouracil plus leucovorin, oxaliplatin, and docetaxel versus fluorouracil or capecitabine plus cisplatin and epirubicin for locally advanced, resectable gastric or gastrooesophageal junction adenocarcinoma (FLOT4): a ra. Lancet 393(10184):1948-1957 (http://www. ncbi.nlm.nih.gov/pubmed/30982686)

50. Tie H, He F, Shen J, Zhang B, Ye M, Chen B et al (2018) Prolonged interval between neoadjuvant chemoradiotherapy and esophagectomy does not benefit the outcome in esophageal cancer: A systematic review and meta-analysis. Dis Esophagus 31:1-9 (http://www.ncbi.nlm.nih.gov/ pubmed/29087451)

51. Shapiro J, van Lanschot JJB, Hulshof MCCM, van Hagen $\mathrm{P}$, van Berge Henegouwen MI, Wijnhoven BPL et al (2015) Neoadjuvant chemoradiotherapy plus surgery versus surgery alone for oesophageal or junctional cancer (CROSS): Long-term results of a randomised controlled trial. Lancet Oncol 16(9):1090-1098 (http://www.ncbi.nlm.nih.gov/ pubmed/26254683)

52. Markar SR, Karthikesalingam A, Penna M, Low DE (2014) Assessment of short-term clinical outcomes following salvage esophagectomy for the treatment of esophageal malignancy: Systematic review and pooled analysis. Ann Surg Oncol 21:922-931 (http://www.ncbi.nlm.nih.gov/ pubmed/24212722)

53. Ariga H, Nemoto K, Miyazaki S, Yoshioka T, Ogawa Y, Sakayauchi T et al (2009) Prospective comparison of surgery alone and chemoradiotherapy with selective surgery in resectable squamous cell 
carcinoma of the esophagus. Int J Radiat Oncol Biol Phys 75(2):348-356

54. Lagarde SM, Maris AKD, De CastroSMM, Busch ORC, Obertop H, Van Lanschot JJB (2007) Evaluation of O-POSSUM in predicting in-hospital mortality after resection for oesophageal cancer. Br J Surg 94(12):1521-1526 (http://www.ncbi.nlm.nih.gov/ pubmed/17929231)

55. Markar SR, Low DE (2013) Physiology, not chronology, dictates outcomes after esophagectomy for esophageal cancer: Outcomes in patients 80 years and older. Ann Surg Oncol 20(3):1020-1026 (http://www.ncbi.nlm.nih.gov/ pubmed/23117475)

56. Handforth C, Clegg A, Young C, Simpkins S, Seymour MT, Selby PJ et al (2015) The prevalence and outcomes of frailty in older cancer patients: a systematic review. Ann Oncol 26(6):1091-1101 (http:// www.ncbi.nlm.nih.gov/pubmed/25403592)

57. Awdeh H, Kassak K, Sfeir P, Hatoum H, Bitar H, Husari A (2015) The SF-36 and 6-minute walk test are significant predictors of complications after major surgery. World J Surg 39(6):1406-1412 (http:// www.ncbi.nlm.nih.gov/pubmed/25651952)

58. Murray $P$, Whiting $P$, Hutchinson SP, Ackroyd R, Stoddard CJ, Billings C (2007) Preoperative shuttle walking testing and outcome after oesophagogastrectomy. Br J Anaesth 99(6):809-811 (http:// www.ncbi.nlm.nih.gov/pubmed/17959592)

59. Pfirrmann $D$, Simon $P$, Mehdorn $M$, Hänsig $M$, Stehr S, Selig L et al (2018) Preconditioning prior to visceral oncological surgery: a paradigm shift in visceral surgery? Chirurg 89(11):896-902 (http:// www.ncbi.nlm.nih.gov/pubmed/30280204)

60. Yanni F, Moyes L, McCaffer C, Carter R (2013) Cardiopulmonary exercise testing as a predictor of complications. Ann RColl Surg Engl 96(1):86-86 (http:// www.ncbi.nlm.nih.gov/pubmed/23484995)

61. Weimann A, Breitenstein S, Breuer JP, Gabor SE, Holland-Cunz S, Kemen M et al (2014) Klinische Ernährung in der Chirurgie: S3-Leitlinie der Deutschen Gesellschaft für Ernährungsmedizin e. V. Chirurg. Apr 85(4):320-326 (http://www.ncbi. nlm.nih.gov/pubmed/24718444)

62. Durkin C, Schisler T, Lohser J (2017) Current trends in anesthesia for esophagectomy. Curr Opin Anaesthesiol 30:30-35 (http://www.ncbi.nlm.nih gov/pubmed/27764049)

63. Findlay JM, Gillies RS, Millo J, Sgromo B, Marshall REK, Maynard ND (2014) Enhanced recovery for esophagectomy: A systematic review and evidence-based guidelines. Ann Surg 259:413-431 (http://www.ncbi.nlm.nih.gov/ pubmed/24253135)

64. Dettling DS, Van der Schaaf M, Blom RLGM, Nollet F, Busch ORC, Van Berge Henegouwen MI (2013) Feasibility and effectiveness of pre-operative inspiratory muscle training in patients undergoing oesophagectomy: a pilot study. Physiother Res Int 18(1):16-26 (http://www.ncbi.nlm.nih.gov/ pubmed/22489016)

65. Van Adrichem EJ, Meulenbroek RL, Plukker JTM, Groen H, Van Weert E (2014) Comparison of two preoperative inspiratory muscle training programs to prevent pulmonary complications in patients undergoing esophagectomy: a randomized controlled pilot study. Ann Surg Oncol 21(7):2353-2360 (http://www.ncbi.nlm.nih.gov/ pubmed/24604584)

66. Le Gall JR (1993) A new simplified acute physiology score (SAPS II) based on a European/north American multicenter study. JAMA 270(24):2957-2963 (http://www.ncbi.nlm.nih. gov/pubmed/8254858)
67. Low DE, Allum W, De Manzoni G, Ferri L, Immanuel A, Kuppusamy M et al (2019) Guidelines for perioperative care in esophagectomy: enhanced recovery after surgery $\left(\right.$ ERAS $\left.^{\circledR}\right)$ society recommendations. World J Surg 43(2):299-330 (http://www. ncbi.nlm.nih.gov/pubmed/30276441)

68. Liao G, Zhao Z, Yang H, Chen M, Li X (2020) Can prognostic nutritional index be a prediction factor in esophageal cancer?: a meta-analysis. Nutr Cancer 72(2):187-193 (https://pubmed.ncbi.nlm. nih.gov/31272238/)

69. Bouchard P, Cools-Lartigue J, Spicer J, Mueller CL, Ferri LE (2017) Endoscopic submucosal dissection for esophageal adenocarcinoma and a north American perspective. Gastroenterology 152(5):S1231

70. Suzuki G, Yamazaki H, Aibe N, Masui K, Sasaki N Shimizu D et al (2018) Endoscopic submucosal dissection followed by chemoradiotherapy for superficial esophageal cancer: choice of new approach. Radiat Oncol 13(1):1-8

71. Gockel I, Hoffmeister A (2018) Endoscopic or surgical resection for gastro-esophageal cancer. Dtsch Arztebl Int 115(31-32):513-519

72. Zeki SS, Bergman JJ, Dunn JM (2018) Endoscopic management of dysplasia and early oesophageal cancer. Best Pract Res Clin Gastroenterol 36-37:27-36. https://doi.org/10.1016/j.bpg.2018. 11.003

73. Pech O, BollschweilerE, MannerH, Leers J, EllC, Hölscher AH (2011) Comparison between endoscopic and surgical resection of mucosal esophageal adenocarcinoma in Barrett's esophagus at two highvolume centers. Ann Surg 254(1):67-72 (http:// www.ncbi.nlm.nih.gov/pubmed/21532466)

74. Reeh M, Nentwich MF, Asani S, Uzunoglu FG, Bockhorn M, Sauter G et al (2015) Locally advanced esophageal carcinoma: is there still a role of surgery alone without neoadjuvant treatment? J Gastrointest Surg 19(4):587-593

75. van Hagen $P$, Hulshof MCCM, van Lanschot JJB, Steyerberg EW, van Henegouwen MIB, Wijnhoven BPL et al (2012) Preoperative chemoradiotherapy for esophageal or junctional cancer. N Engl J Med 366(22):2074-2084. https://doi.org/10.1056/ NEJMoa1112088

76. Al-Batran S-E, Hartmann JT, Hofheinz R, Homann N, Rethwisch V, Probst S et al (2008) Biweekly fluorouracil, leucovorin, oxaliplatin, and docetaxel(FLOT) for patients with metastatic adenocarcinoma of the stomach or esophagogastric junction: a phase II trial of the Arbeitsgemeinschaft Internistische Onkologie. Ann Oncol 19(11):1882-1887 (http:// www.ncbi.nlm.nih.gov/pubmed/18669868)

77. Straatman J, Van DerWielen N, Cuesta MA, DaamsF, Roig Garcia J, Bonavina L et al (2017) Minimally invasive versus open esophageal resection. Ann Surg 266(2):232-236

78. MarietteC,MarkarS, Dabakuyo-YonliTS,MeunierB, Pezet D, Collet D et al (2019) Health-related quality of life following hybrid minimally invasive versus open esophagectomy for patients with esophageal cancer, analysis of a multicenter, openlabel, randomized phase III controlled trial: the MIRO trial. Ann Surg 271(6):1023-1029

79. Levinsky NC, Wima K, Morris MC, Ahmad SA, Shah SA, Starnes SL et al (2019) Outcome of delayed versus timely esophagectomy after chemoradiation for esophageal adenocarcinoma. J Thorac Cardiovasc Surg. http://www.ncbi.nlm. nih.gov/pubmed/31767364. Zugegriffen: 4. Jan. 2020
80. Lorimer PD, Motz BM, Boselli DM, Reames MK Hill JS, Salo JC (2019) Quality improvement in minimally invasive esophagectomy: outcome improvement through data review. Ann Surg Oncol 26(1):177-187. https://doi.org/10.1245/ s10434-018-6938-z 
Hier steht eine Anzeige.

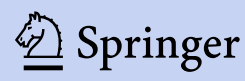

